\title{
BMJ Open Assessing the benefits on quality of life of a multicomponent intervention for fibromyalgia syndrome in primary care: patients' and health professionals' appraisals: a qualitative study protocol
}

Victoria Mailen Arfuch (D) ,,2 Rosa Caballol Angelats,, ${ }^{3,4}$ Carina Aguilar Martín,, Noèlia Carrasco-Querol, ${ }^{1}$ Maria Cinta Sancho Sol, ${ }^{1,4}$ Gemma González Serra, ${ }^{1,4}$ Immaculada Fusté Anguera, ${ }^{3,4}$ Alessandra Queiroga Gonçalves, ${ }^{1,6}$ Anna Berenguera (iD 2,7

To cite: Arfuch VM, Caballol Angelats R, Aguilar Martín C, et al. Assessing the benefits on quality of life of a multicomponent intervention for fibromyalgia syndrome in primary care: patients' and health professionals appraisals: a qualitative study protocol. BMJ Open 2020;10:e039873. doi:10.1136/ bmjopen-2020-039873

- Prepublication history for this paper is available online. To view these files, please visit the journal online (http://dx.doi. org/10.1136/bmjopen-2020039873).

Received 28 April 2020 Revised 07 October 2020 Accepted 23 October 2020

Check for updates

(C) Author(s) (or their employer(s)) 2020. Re-use permitted under CC BY-NC. No commercial re-use. See rights and permissions. Published by BMJ.

For numbered affiliations see end of article.

Correspondence to

Dr Anna Berenguera;

aberenguera@idiapjgol.org

\section{ABSTRACT}

Introduction Fibromyalgia syndrome (FMS) is a complex condition still scarcely understood and with ambiguity when prescribing treatment. Both patients and healthcare providers can supply valuable information for the development of new treatment strategies. The qualitative narrative analysis of participant's accounts is potentially helpful to reveal new insights about their opinions, needs, and experiences and, consequently, to model healthcare interventions accurately. International treatment guidelines suggest a promising future for multicomponent intervention (MI) approaches for FMS. This study aims to assess the benefits of a Ml for patients with FMS in the context of primary care (PC) in Terres de L'Ebre, Catalonia (Spain). Furthermore, it is intended to detect the overall perception of effectiveness and to understand patients' lived experience and its impact on the quality of life.

Method and analysis Qualitative research from a socioconstructivism paradigm perspective and a Hermeneutic Phenomenological method. For data collection, four focus group discussions (FGDs) of 8-12 people (2 FGDs of patients and 2 of professionals) and 10-12 key informant interviews with the participants in the Ml group will be carried out. All the information will be recorded and verbatim transcribed to perform an interpretive thematic analysis.

Ethics and dissemination This study protocol has been approved by the Clinical Research Ethics Committee from the IDIAPJGol Institute, on 25 April 2018 (code P18/068), according to the Declaration of Helsinki/Tokyo. All participants will receive oral/written information about the study, and they will be required to sign an informed consent sheet. Data anonymity will be guaranteed. Dissemination will be carried out through publications in scientific journals, presentations in academic meetings, workshops and through the local and national media. Trial registration number ClinicalTrials.gov: NCT04049006; Pre-results.

\section{INTRODUCTION}

Fibromyalgia is a long-term condition, potentially disabling characterised especially by an atypical perception of pain. This syndrome disrupts patients' biopsychosocial universe

\section{Strengths and limitations of this study}

- This study will provide information on barriers and facilitators about the multicomponent intervention (MI) programme for fibromyalgia syndrome to design more flexible treatment strategies adapted to patients' health needs.

- The results of this study will help to improve the contents of a Ml considering patients' and professionals' opinions and the available resources in primary care.

- In-depth patient interviews will contribute to a richer understanding of their health needs, lived experiences and the subjective benefits of the MI to provide accurate and higher quality health services.

- A pre-implementation phase through qualitative methodology has not been conducted before the Ml started.

- The results of this study will not be generalisable to other contexts due to the limitations of the method.

compromising personal and occupational aspects, plans and relationships. ${ }^{1}$ Consequently, global health status and quality of life (QOL) could be distorted. ${ }^{2}$

Fibromyalgia syndrome (FMS) is a complex condition associated with several symptoms and comorbidities, such as rheumatological and psychiatric disorders. ${ }^{3}$ FMS diagnosis is based on clinical criteria where the most frequent manifestations are: generalised musculoskeletal pain, fatigue, sleep disturbance, gastrointestinal disorders, psychological distress which can trigger anxiety and depression, loss of functioning, low tolerance to physical effort, migraines and dizziness. ${ }^{4-6}$

The worldwide prevalence of FMS in the general population is estimated between 
$0.2 \%$ and $6.6 \%$ and, $2.45 \%$ on average in Spain. ${ }^{78}$ It is usually diagnosed in middle-aged people, ${ }^{29}$ and it is more frequent in women with a prevalence ranged between $2.4 \%$ and $6.8 \%$ and with a female/male ratio of $21: 1 .^{10}$ Although this difference in prevalence between women and men cannot be fully explained yet, studies suggest that these rates are overestimated for women and underestimated for men due to diagnostic criteria bias. ${ }^{11}{ }^{12}$ Moreover, another study identifies the existence of gender bias in healthcare towards patients with chronic pain, which helps to demystify the FMS as a predominantly female condition due to intrinsic factors. ${ }^{13}$

Although FMS is classified as a central sensitivity syndrome, ${ }^{14}{ }^{15}$ its pathogenesis, aetiology and evolution are still medically unexplained. Some experts suggest an interaction between neurophysiological, genetic, epigenetics and psychosocial factors. ${ }^{16-19}$ This knowledge gap complexes and delays the diagnosis process, discrediting the authenticity of the fibromyalgia, and generating ambivalence about the provision of healthcare. ${ }^{20} 21$

Diverse treatment strategies have been tested focusing on severity symptom management, QOL improvement and the prevention of chronicity. Physical exercise, psychological approaches such as cognitive-behavioural therapy (CBT), health education and complementary medicine have demonstrated some clinical improvement in symptom control. ${ }^{22-27}$ Nevertheless, the evidence remains uncertain, and there is scope for further research to determine the best treatment option. In this context, users' and professionals' opinions become a strategic resource for the design of treatments tailored to health needs. A qualitative analytic approach to understanding their point of views and necessities is a valuable method which reveals novel insights about how people make sense of this condition and how they experience treatments. ${ }^{28}$

There are few published qualitative studies about the effectiveness of interventions in patients suffering from FMS. According to the evidence, qualitative methodology is a useful approach to assess in-depth participants' treatment experience and detect patients' global impression of change regarding pain management, functionality and QOL. ${ }^{29-34}$ However, authors acknowledged that it would be important to include the clinicians' appraisals as well. ${ }^{30}$

Multidisciplinary interventions that combine pharmacological and non-pharmacological treatments have shown to be effective in some clinical trial studies after 3-12 months of follow-up. ${ }^{30}{ }^{35-39}$ A recent study, based on a comprehensive 8-week group-based multidisciplinary rehabilitation programme that included CBT and graduated activity training, showed significant improvements in health-related QoL at the end of the intervention, at 6 months and one year of follow-up. ${ }^{40}$ Regarding the Spanish population, a study of similar characteristics was conducted in the Basque Country in 2012, about a 6-week interdisciplinary treatment in the hospital context. This programme that combined psychological, medical, educational and physiotherapeutic components demonstrated significant improvements in QOL, physical function and pain at a 6-month follow-up. Although these results were maintained at 12 months, they were not compared with a control group, nor were they studied in the context of primary care $(\mathrm{PC}){ }^{41}$

Multicomponent intervention (MI) programmes have been recommended in international treatment guidelines. ${ }^{42-45}$ Nevertheless, this intervention strategy for FMS is in its infancy in the usual clinical care ${ }^{46}$ into the Spanish public health sector. In Catalonia, and since 2016, 18 accredited units specialised in Central Sensitivity Syndromes located in PC centres and hospitals ${ }^{47}$ provide multidisciplinary healthcare to patients with $\mathrm{FMS}^{48}$

This study aims to search out, through qualitative research methodology and from patients with FMS and healthcare providers' points of view, the overall perception of effectiveness about a MI and its benefits on patients' QOL. Moreover, the specific objectives of the study are:

- To assess the subjective benefits on patients' functional impact, mood and pain management.

- To assess professionals' experience about the MI and their appraisal regarding the benefits of the programme for patients' health.

- To detect improvable aspects of the MI, based on the opinions and experiences of participants (users and professionals), in order to standardise the programme so that it can be replicated in other health settings.

- To explore the subjective benefits and the impact of the MI on patients' daily life and to understand the structure of their lived experiences.

This MI programme is provided within the $11 \mathrm{PC}$ centres of the Gerència Territorial Terres de L'Ebre of the Catalan Institute of Health. It consists of a 3-month group programme combining: health education, physical activity and CBT. The purpose of this proposal is to promote patients' QOL by increasing control over their health in order to improve it.

It is expected that the results will support and extend the results of the randomized clinical trial (RCT) from which this project arises (ClinicalTrials.gov: NCT04049006). ${ }^{49}$ The contents of each MI session and other details of the design are fully described in the published protocol of the RCT study. ${ }^{50}$

\section{METHOD \\ Design}

This protocol has been drafted based on the literature review and following the protocol guidance for qualitative research of the Health Research Authority. ${ }^{51}$ Furthermore, this study will be reported following the Consolidated Criteria for Reporting Qualitative Research $^{52}$ and the Standards for Reporting Qualitative Research: A Synthesis of Recommendations. ${ }^{53}$ Finally, the evaluation of the intervention will be founded on the constructs and guidelines suggested by experts of implementation research. ${ }^{5455}$ 
For the design of this study, interpretive qualitative research will be carried out based on the theoretical framework of the socioconstructivism paradigm ${ }^{56}$ and a Hermeneutic Phenomenological method. ${ }^{57}$ This perspective aims to answer what are the meanings that give sense to our individual and collective existence, and how they come to be constituted. ${ }^{58}$ In this way, the narrative occurs and evolves in the process of social interaction, where the discourse does not pre-exist but is constructed in the intersubjective setting. Therefore, socially constructed narrative realities give significance and structure to our existence, and the interpretation of this experience challenges the way of being in a particular context. ${ }^{59}$ This study design is also accurate since it incorporates the preunderstanding of the researcher as part of the experience and so it is not necessary bracketing particular beliefs about the phenomena in order to explore it.

The multiperspective research approach collects data from informants with different positioning regarding the study phenomenon, expanding not only the narrative themes of the accounts but also enriching the understanding of the structure and dynamics of interaction between them. ${ }^{60} 61$

\section{Intervention strategy}

The MI consists of a 12-week group programme of 2 hours weekly combining: 7 health education instructions delivered by a general practitioner and a nurse, 11 pieces of training on physical activity delivered by a physiotherapist, and 7 interventions of CBT delivered by a psychologist.

Regarding the contents of the programme, the health education component includes an introduction to multicomponent therapy, neurophysiology and pharmacology of pain, techniques of postural hygiene, nutrition, insomnia management, memory and sexuality. The physical activity component focuses on breathing and relaxation, stretching, strength/joint and coordination exercises, and the 6 min walking test. Finally, the psychological component works on CBT strategies, pain and attention management, mood and emotional management, strategies for coping with difficulty, and the planning of goals and pleasant activities.

This MI programme aims to: the patients' acquisition of knowledge, skills and attitudes for the daily management of the FMS; the improvement of their physical condition; the relief of their emotional distress, and to provide skills to overcome psychological difficulties.

\section{Participants and setting}

This study will include patients who have had a minimum of participation in the MI group and PC professionals involved in some stage of the programme. All participants will be recruited from any of the 11 PC centres belonging to the Gerència Territorial Terres de l'Ebre (Catalan Health Institute).

The inclusion criteria for patients are: have a clinical diagnosis of FMS (International Classification of Diseases-10 codes: M79.0, M79.7), being over 18 years, be able to communicate (orally and written) effectively in Catalan or Spanish, have a phone number, have participated in a minimum of $75 \%$ of the intervention programme (equal to or more than 9 out of 12 sessions), accept participation in the qualitative study, sign the informed consent. On the other hand, the exclusion criteria involve: having an actively psychotic episode, having an intellectual impairment, having severe depression and personality disorder, registering auto/hyperaggressive behaviour, detecting use of psychoactive substances, having not attended to a minimum of $75 \%$ of the MI group sessions (less than 9 out of 12 sessions), not sign the informed consent.

The criteria for professional recruitment include sign the informed consent sheet, have participated in some stage of the MI in the last 6 months, not having declared conflicts of interest.

Group and individual interviews with patients and professionals will be carried out in a PC centre from Terres de L'Ebre area. Sound insulation criteria will be considered to guarantee the confidentiality of the information.

\section{Patient and public involvement}

Patients or the public will not be involved in the design or conducting, or reporting, or dissemination plans of our research.

\section{Sampling and recruitment}

Theoretical sampling will be used to achieve maximum discursive variability. First, two lists of possible candidates among patients will be drafted based on time criteria (up to $<6$ months of follow-up after the intervention and, between $\geq 6$ and 12 months of follow-up) in order to assess the perception of the benefits in the short and medium-long term. Second, those patients who accomplish with a minimum attendance of $75 \%$ to the intervention group will be included in the recruitment sample. Then, criteria based on gender, age, working status and geographical area will be applied to draft a list of possible candidates. Finally, patients will be contacted by phone, and only those who accept participating in the qualitative study will be included in the final data collection sample. Regarding PC professionals, general practitioners and nurses of different genders, age groups, geographical areas of work will be included.

Additionally, and in order to understand the reasons for MI dropout and the possible lack of adherence, 4-6 phone calls will be done to patients who have been recruited to participate in the study and have signed the informed consent but who, finally, did not start the programme or wanted to leave it early. Members of the research group will carry out these phone calls according to the data protection low.

Informants will be invited to participate in the study through telephone contact. They will be informed about the characteristics and objectives of the study, the audiovideo recording of the interviews, and they will be guaranteed with the anonymity of the data management. 


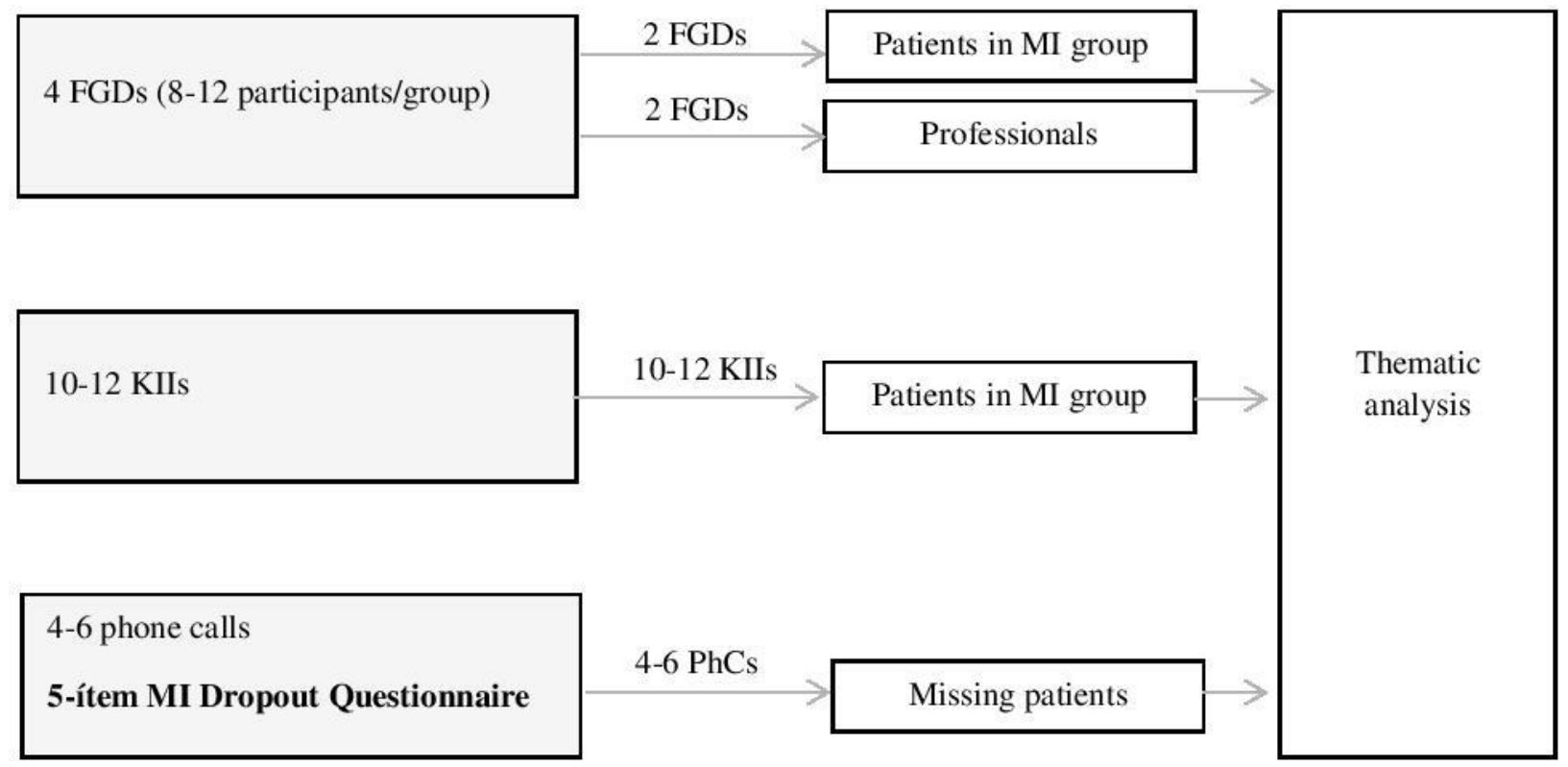

Figure 1 Flow diagram of the qualitative study. FGD, focus group discussions; KIl, key informant interview; MI, multicomponent intervention; PhCs, phone calls.

People who have participated in both study groups because of an ethical requirement (first control and then intervention group) will be recruited for some of the individual interviews. Although this subsample cannot be included in the quantitative study for methodological reasons, they could provide valuable information for the qualitative analysis since they will have experienced both treatment interventions.

\section{Data collection}

Data collection includes four focus group discussions (FGDs) of 8-12 people (2 FGDs of patients and 2 of professionals) and 10-12 key informant interviews (KIIs) with patients that have participated in the MI group. FGDs and KIIs will be carried out through different samples. When necessary, additional discussion groups and personal interviews will be conducted to achieve discourse saturation. Data collection is expected to be complete by January 2021. Figure 1 describes the design and methodology of data collection.

The FGDs and KIIs will be audio and video recorded, prior informed consent, and will be literally and systematically transcribed. Identifying data of the informants in the transcripts will be anonymised.

An experienced moderator will conduct the FGDs using a semistructured guide. A comoderator and an observer will also participate as part of the research team. The FGDs are expected to last no more than $95 \mathrm{~min}$. The guide will cover the following topics: the overall opinion about the MI effectiveness, an evaluation of the elements of the framework (time, space, professionals and beneficiaries), improvable aspects, and the benefits of the intervention on patients' $Q O L$.

KIIs will be delivered by an experienced qualitative researcher and will be based on open-ended structured questions. The objective is to assess the patients' personal lived experience in the intervention programme and exploring their subjective impact. The interviews are expected to last no more than $65 \mathrm{~min}$.

The question protocols for the FGDs and the KIIs will include open-ended questions aimed to cover the research objectives: patients' experiences during the MI and the subjective benefits, its impact on QOL and daily life, patients' and professionals' overall perception of effectiveness, and barriers and facilitators on the intervention implementation.

\section{Data analysis}

A thematic analysis will be carried out to identify, interpret and report patterns (themes) within data. First, literal transcripts of the audio recordings will be included in a text corpus according to a published guide, which enlists the steps for thematic analysis. ${ }^{62}$ Secondly, successive readings of the text corpus will be completed as well as the formulation of pre-analytical intuitions. Thirdly, the coding process will be delivered, organising the data into the most basic meaningful elements (codes). Consecutively, the emergent themes from the interpretative analysis will be registered and clustered where appropriate under superordinate themes or salient phrases. In this way, all the relevant coded data extracts will be organised within the identified themes to catch the essential attributes of the participants' accounts. These themes will be reviewed and named checking the existence of coherent patterns. Finally, a report will be drafted writing up the results of the analysis and the interpretation of it.

Furthermore, a triangulation process of this first thematic analysis will be fulfilled between two or more members of the research team. ${ }^{63}$ Discrepancies in the data analysis triangulation process will be resolved primarily through consensus. In case this is not achieved, the majority criteria will be implemented, if applicable. 
If none of these strategies worked, the external expert opinion would be appealed to solve the struggle. Nevertheless, an attempt will be made to reach agreements between researchers that include all points of view, fulfilling the criterion of internal coherence. The meanings extract from the triangulation will be interpreted, and an explanatory framework will be created with the contributions of each type of informant (users and professionals participating in the FGDs and the KIIs).

\section{DISCUSSION}

FMS has become a challenge for health providers due to its heterogeneity, unclear aetiopathogenesis and the particularities of the patients' profile. ${ }^{20}$ Faced with this scenario, it is vital to incorporate a holistic perspective of all those involved in the development and implementation of treatment strategies, including people suffering from this condition.

From a methodological perspective and according to the Medical Research Council, the qualitative approach provides relevant information for the validation and modelling of new intervention programmes in order to effectively implement them in the real world and to identify and understand the main barriers. ${ }^{6465}$ Moreover, the communication with stakeholder groups informs if the intervention addresses the priority issues for the people involved and uses appropriate, feasible and acceptable methods. ${ }^{66}$

Accordingly, the qualitative assessment is expected to reveal key details of the MI implementation, from patients' and professionals' points of view involved. This information will allow adjusting the setting elements (timeline, number of participants in the group), therapeutic components and thematic contents that cover patients' health needs. Additionally, the results of this study will likely improve the adherence to the MI, the adequacy of health centres and the impact on family members. Consequently, healthcare services for FMS, provided on the recently established units specialised in Central Sensitivity Syndromes in the area, will increase their effectiveness and efficiency. As well, this programme will be hopefully replicated and considering as an intervention model in other healthcare systems.

A triangulation technique will be implemented to achieve complex analysis. The multiperspective qualitative approach can also help to study relationships and dynamics between patients and professionals and to explore similarities and differences in their perceptions.

Furthermore, in-depth patient interviews will contribute to a richer understanding of their real health needs, lived experiences and the subjective benefits of the MI. This information is crucial for providing accurate and highquality health services and collaborates with FMS acceptance and self-management.

Even though the qualitative approach is considered a weak method to achieve reliable evidence, it can also be successful in seeking little studied and controversial topics related to FMS. Patients' experiences could guide researchers on the path to resolve the FMS dilemma. Understanding the experience of illness and how this syndrome impacts at different levels such as work, social, physical, psychological and sexual functionality could help to determine how to approach it from the healthcare services to improve patients' QOL and overcome its legitimacy debate.

Attending the limitations of the qualitative methodology, the results of this study will not be generalisable to other contexts. Moreover, due to the nature of selfreported data, the results could be affected by memory, group effect, overestimation or underestimation, selection, or attribution biases. In addition to the intrinsic limitations of the method, this qualitative study also presents shortcomings. Firstly, any pre-implementation phase was conducted. Secondly, inclusion criteria in the FGDs and KIIs will be limited only to those people who agree to participate in the qualitative study so that they could have a greater interest and a more positive perception of the MI. Another limitation is that, initially, no external expert in qualitative methodology will be included in the triangulation analysis process to avoid investigator bias. It will only be considered in case of major discrepancies between the researchers. Finally, there will be no FGDs according to sex due to the lack of a necessary sample of male patients.

Regarding the healthcare context, PC services could address this issue, providing support to their patients, preventing health decline, and motivating these people to feel empowered in coping with FMS. Effective management actions in PC could reduce the costs of specialised professional care, overprovision and overmedication, avoiding system overload, and providing more holistic care to the needs of patients with FMS.

Equally important, new health policies and specialised training programmes that increase knowledge and sensitisation of health providers are needed to resolve fibromyalgia's lack of recognition and stigma based on social construction in the Spanish context. ${ }^{67}$ A comprehensive assessment of the FMS symptomatology and its impact on the QoL of patients should be part of the usual clinical care. $^{68}$

\section{ETHICS AND DISSEMINATION}

The Clinical Research Ethics Committee from the Fundació Institut Universitariper a la recerca a l'Atenció Primària de Salut Jordi Gol $i$ Gurina (IDIAPJGol), has approved this study protocol on 25/04/2018 (code P18/068), according to the Declaration of Helsinki/Tokyo. All participants will receive oral/written information about the study, and they will be required to sign an informed consent sheet. Data anonymity will be guaranteed. Dissemination will be carried out through publications in scientific journals, presentations in academic meetings, workshops and through the local and national media. 
Author affiliations

${ }^{1}$ Unitat de Suport a la Recerca Terres de I'Ebre, IDIAP Jordi Gol, Barcelona, Catalunya, Spain

${ }^{2}$ Department of Pediatrics, Obstetrics and Gynecology, and Preventive Medicine, Universitat Autonoma de Barcelona, Barcelona, Catalunya, Spain

${ }^{3}$ Equip d'Atenció Primària Tortosa Est, Institut Català de la Salut, Tortosa, Catalunya, Spain

${ }^{4}$ Unitat d'Expertesa en Sindromes de Sensibilització Central Terres de l'Ebre, Institut Català de la Salut, Tortosa, Catalunya, Spain

'Unitat d'Avaluació, Direcció d'Atenció Primària Terres de l'Ebre, Institut Català de la Salut, Tortosa, Catalunya, Spain

${ }^{6}$ Unitat Docent de Medicina de Família i Comunitària Tortosa-Terres de L'Ebre, Institut Català de la Salut, Tortosa, Catalunya, Spain

${ }^{7}$ Central Research Unit, IDIAP Jordi Gol, Barcelona, Catalunya, Spain

\section{Twitter Anna Berenguera @ABerenguera}

Acknowledgements All authors thank the contributions of the ICS, especially to the Gerència Territorial ICS Terres de l'Ebre, la Direcció d'Atenció Primària de Terres de l'Ebre, and the Unitat de Sistemes d' informació de la Gerència Territorial Terres de l'Ebre. Likewise, the participation and support of all healthcare and nonhealthcare professionals, who collaborate in the implementation of this study and data collection, are appreciated.

Contributors VMA, AB, CAM, RCA and AQG participated in the design of the study. VMA wrote the draft versions with the review of all authors. All authors are involved in the development of the general project and the RCT study from which this qualitative study is related.

Funding This project received a research grant from the Instituto de Salud Carlos III (ISCIII) awarded in 2018 with reference PI1800943. This grant belongs to the Health Strategy Action 2017-2020 within the National Research Program; the Technical, Scientific and Innovation Research National Plan 2017-2020; and it is also co-funded by the European Union ERDF funds (European Regional Development Fund). Furthermore, a Predoctoral grant for training in health research was obtained in 2019 from the ISCIII (reference Fl19/00254). Finally, this project was funded with a scholarship from Dr Ferran Foundation in 2017.

Disclaimer The funders financed the project but will not participate in any phase of the investigation. The funders will not have any authority over any of the activities of the project.

Competing interests None declared.

Patient and public involvement Patients and/or the public were not involved in the design, or conduct, or reporting, or dissemination plans of this research.

Patient consent for publication Not required.

Provenance and peer review Not commissioned; externally peer reviewed.

Open access This is an open access article distributed in accordance with the Creative Commons Attribution Non Commercial (CC BY-NC 4.0) license, which permits others to distribute, remix, adapt, build upon this work non-commercially, and license their derivative works on different terms, provided the original work is properly cited, appropriate credit is given, any changes made indicated, and the use is non-commercial. See: http://creativecommons.org/licenses/by-nc/4.0/.

\section{ORCID iDs}

Victoria Mailen Arfuch http://orcid.org/0000-0001-5935-6907

Anna Berenguera http://orcid.org/0000-0002-0889-2002

\section{REFERENCES}

1 Wuytack F, Miller P. The lived experience of fibromyalgia in female patients, a phenomenological study. Chiropr Man Therap 2011;19:22

2 Mas AJ, Carmona L, Valverde M, et al. Prevalence and impact of fibromyalgia on function and quality of life in individuals from the general population: results from a nationwide study in Spain. Clin Exp Rheumatol 2008;26:519-26.

3 Lichtenstein A, Tiosano S, Amital H. The complexities of fibromyalgia and its comorbidities. Curr Opin Rheumatol 2018;30:94-100.

4 Wolfe F, Smythe HA, Yunus MB, et al. The American College of rheumatology 1990 criteria for the classification of fibromyalgia. Report of the multicenter criteria Committee. Arthritis Rheum 1990;33:160-72.
5 Wolfe F, Clauw DJ, Fitzcharles M-A, et al. Fibromyalgia criteria and severity scales for clinical and epidemiological studies: a modification of the ACR preliminary diagnostic criteria for fibromyalgia. J Rheumatol 2011;38:1113-22.

6 Wolfe F, Clauw DJ, Fitzcharles M-A, et al. 2016 revisions to the 2010/2011 fibromyalgia diagnostic criteria. Semin Arthritis Rheum 2016;46:319-29.

7 Seoane-Mato D, Sánchez-Piedra C, Silva-Fernández L, et al. Prevalence of rheumatic diseases in adult population in Spain (EPISER 2016 study): aims and methodology. Reumatol Clin 2019;15:90-6.

8 Cabo-Meseguer A, Cerdá-OImedo G, Trillo-Mata JL. Fibromyalgia: prevalence, epidemiologic profiles and economic costs. Med Clin 2017;149:441-8.

9 Villanueva VL, Valía JC, Cerdá G, et al. Fibromialgia: diagnóstico Y tratamiento. El estado de la cuestión. Rev Soc Esp Dolor 2004;11.

10 Marques AP, Santo AdeSdoE, Berssaneti AA, et al. Prevalence of fibromyalgia: literature review update. Rev Bras Reumatol Engl Ed 2017;57:356-63.

11 Wolfe F, Walitt B, Perrot S, et al. Fibromyalgia diagnosis and biased assessment: sex, prevalence and bias. PLoS One 2018;13:e0203755

12 Häuser W, Sarzi-Puttini P, Fitzcharles M-A. Fibromyalgia syndrome: under-, over- and misdiagnosis. Clin Exp Rheumatol 2019;37 Suppl 116:S90-7.

13 Samulowitz A, Gremyr I, Eriksson E, et al. "Brave Men" and "Emotional Women": A Theory-Guided Literature Review on Gender Bias in Health Care and Gendered Norms towards Patients with Chronic Pain. Pain Res Manag 2018;2018:1-14.

14 Fleming KC, Volcheck MM. Central sensitization syndrome and the initial evaluation of a patient with fibromyalgia: a review. Rambam Maimonides Med J 2015;6:e0020-12.

15 Fitzcharles MA, Ste-Marie PA, Pereira JX. Md for Canadian fibromyalgia guidelines Committee. fibromyalgia: evolving concepts over the past 2 decades. CMAJ 2013;185:E645-51.

16 Gran JT, Tore Gran J. The epidemiology of chronic generalized musculoskeletal pain. Best Pract Res Clin Rheumatol 2003;17:547-61.

17 Abeles AM, Pillinger MH, Solitar BM, et al. Narrative review: the pathophysiology of fibromyalgia. Ann Intern Med 2007;146:726-34

18 Malatji BG, Meyer H, Mason S, et al. A diagnostic biomarker profile for fibromyalgia syndrome based on an NMR metabolomics study of selected patients and controls. BMC Neurol 2017;17:88.

19 D'Agnelli S, Arendt-Nielsen L, Gerra MC, et al. Fibromyalgia: genetics and epigenetics insights may provide the basis for the development of diagnostic biomarkers. Mol Pain 2019;15:1744806918819944.

20 Helfenstein M, Goldenfum MA, Siena CAF. Fibromyalgia: clinical and occupational aspects. Rev Assoc Med Bras 2012;58:358-65.

21 Burke KG. Year in Review-2019. J Nurses Prof Dev 2019;35:303-4.

22 Bush AJ, Webber SC, Richards RS. Resistance exercise training for fibromyalgia. Cochrane Database Syst Rev 2013;12:CD0108.

23 Bernardy K, Klose P, Bush AJ, et al. Cognitive behavioral therapies for fibromyalgia. Cochrane Database Syst Rev 2013;3:CD00979.

24 Luciano JV, Guallar JA, Aguado J, et al. Effectiveness of group acceptance and commitment therapy for fibromyalgia: a 6-month randomized controlled trial (EFFIGACT study). Pain 2014;155:693-702.

25 Aman MM, Jason Yong R, Kaye AD, et al. Evidence-Based nonpharmacological therapies for fibromyalgia. Curr Pain Headache Rep 2018;22:33

26 Prabhakar A, Kaiser JM, Novitch MB, et al. The role of complementary and alternative medicine treatments in fibromyalgia: a comprehensive review. Curr Rheumatol Rep 2019;21:14.

27 Baranowsky J, Klose P, Musial F, et al. Qualitative systemic review of randomized controlled trials on complementary and alternative medicine treatments in fibromyalgia. Rheumatol Int 2009;1:30.

28 McMahon L, Murray C, Simpson J. The potential benefits of applying a narrative analytic approach for understanding the experience of fibromyalgia: a review. Disabil Rehabil 2012;34:1121-30.

29 Hyland ME, Hinton C, Hill C, et al. Explaining unexplained pain to fibromyalgia patients: finding a narrative that is acceptable to patients and provides a rationale for evidence based interventions. Br J Pain 2016;10:156-61.

30 Bourgault $\mathrm{P}$, Lacasse A, Marchand S, et al. Multicomponent interdisciplinary group intervention for self-management of fibromyalgia: a mixed-methods randomized controlled trial. PLoS One 2015;10:e0126324

31 Kashikar-Zuck S, Tran ST, Barnett K, et al. A qualitative examination of a new combined cognitive-behavioral and neuromuscular training intervention for juvenile fibromyalgia. Clin J Pain 2016;32:70-81. 
32 Miranda NACG, Berardinelli LMM, Sabóia VM, et al. Interdisciplinary care praxis in groups of people living with fibromyalgia. Rev Bras Enferm 2016;69:1115-23.

$33 \mathrm{Sim}$ J, Madden S. Illness experience in fibromyalgia syndrome: a metasynthesis of qualitative studies. Soc Sci Med 2008;67:57-67.

34 Oliveira JPR, Berardinelli LMM, Cavaliere MLA, et al. The routines of women with fibromyalgia and an interdisciplinary challenge to promote self-care. Rev Gauch Enferm2019;40:e20180411.

35 Giusti EM, Castelnuovo G, Molinari E. Differences in multidisciplinary and interdisciplinary treatment programs for fibromyalgia: a mapping review. Pain Res Manag 2017;2017:1-19.

36 Ollevier A, Vanneuville I, Carron P. A 12-week multicomponent therapy in fibromyalgia improves health but not in concomitant moderate depression, an exploratory pilot study. Disabil Rehabil 2019;0:1-8.

37 Martín J, Torre F, Padierna A, et al. Six-and 12-month follow-up of an interdisciplinary fibromyalgia treatment programme: results of a randomised trial. Clin Exp Rheumatol 2012;30:103-11.

38 Saral I, Sindel D, Esmaeilzadeh S, et al. The effects of long- and short-term interdisciplinary treatment approaches in women with fibromyalgia: a randomized controlled trial. Rheumatol Int 2016;36:1379-89.

39 Stein KF, Miclescu A. Effectiveness of multidisciplinary rehabilitation treatment for patients with chronic pain in a primary health care unit. Scand J Pain 2013;4:190-7.

40 Jacobs $\mathrm{H}$, Bockaert M, Bonte J, et al. The impact of a group-based multidisciplinary rehabilitation program on the quality of life in patients with fibromyalgia: results from the QUALIFIBRO study. $J$ Clin Rheumatol 2019. doi:10.1097/RHU.0000000000001120. [Epub ahead of print: 19 Jun 2019].

41 Martín J, Torre F, Padierna A, et al. Six-and 12-month follow-up of an interdisciplinary fibromyalgia treatment programme: results of a randomised trial. Clin Exp Rheumatol 2012;30:S103-11.

42 Thieme K, Mathys M, Turk DC. Evidenced-Based guidelines on the treatment of fibromyalgia patients: are they consistent and if not, why not? have effective psychological treatments been overlooked? J Pain 2017:18:747-56.

43 Arnold B, Häuser W, Arnold M, et al. Multicomponent therapy of fibromyalgia syndrome. Der Schmerz 2012;26:287-90.

44 Nüesch E, Häuser W, Bernardy K, et al. Comparative efficacy of pharmacological and non-pharmacological interventions in fibromyalgia syndrome: network meta-analysis. Ann Rheum Dis 2013;72:955-62.

45 Papadopoulou D, Fassoulaki A, Tsoulas C, et al. A meta-analysis to determine the effect of pharmacological and non-pharmacological treatments on fibromyalgia symptoms comprising OMERACT-10 response criteria. Clin Rheumatol 2016;35:573-86.

46 de SD. Guia per a $L$ avaluació de la fibromi lgia I de la síndrome de fatiga crònica. Generalitat de Catalunya, 2017.

47 Generaliat de Catalunya, Agència de Qualitat i Avaluació Sanit ries de Catalunya. Evaluació $Y$ abordaje de la fibromialgia $Y$ síndrome de fatiga crónica, 2017. Available: http://aquas.gencat.cat/web/.content/ minisite/aquas/publicacions/2017/evaluacion_fibromialgia_SFC_ aquas2017es.pdf [Accessed 12 Feb 2020].

48 Generalitat de Catalunya. Pla operatiu d'atenció a les persones afectades per les síndromes de senzibilització central: fibromi Igia, síndrome de fatiga crònica i síndrome de sensibilitat química múltiple, 2016. Available: https://canalsalut.gencat.cat/ca/salut-a-z/f/ fibromialgia/ [Accessed 12 Feb 2020].

49 Caballol Angelats R, Gonçalves AQ, Aguilar Martín C, et al. Effectiveness, cost-utility, and benefits of a multicomponent therapy to improve the quality of life of patients with fibromyalgia in primary care: a mixed methods study protocol. Medicine 2019;98:e17289.

50 Caballol Angelats R, Gonçalves AQ, Aguilar Martín C, et al. Effectiveness, cost-utility, and benefits of a multicomponent therapy to improve the quality of life of patients with fibromyalgia in primary care: a mixed methods study protocol. Med 2019;98.

51 NHS Health Research Authority. Protocol guidance and template for use in qualitative research. Available: https://www.hra.nhs.uk/ planning-and-improving-research/research-planning/protocol/ [Accessed $14 \mathrm{Feb} 2020$ ].

52 Tong A, Sainsbury P, Craig J. Consolidated criteria for reporting qualitative research (COREQ): a 32-item checklist for interviews and focus groups. Int J Qual Health Care 2007;19:349-57.

53 O'Brien BC, Harris IB, Beckman TJ, et al. Standards for reporting qualitative research: a synthesis of recommendations. Acad Med 2014;89:1245-51.

54 Brownson R, Colditz G, Proctor E. Dissemination and implementation research in health. New York, USA: Oxford University Press, 2012.

55 Proctor EK, Landsverk J, Aarons G, et al. Implementation research in mental health services: an emerging science with conceptual, methodological, and training challenges. Adm Policy Ment Health 2009;36:24-34

56 Wilson HJ. The myth of objectivity: is medicine moving towards a social constructivist medical paradigm? Fam Pract 2000;17:203-9.

57 Tindall L. Book review from Smith JA, flower P, Larkin M (2009), interpretative phenomenological analysis: theory, method and research. Qual Res Psychol 2009;6:346-7.

58 Laverty SM, Phenomenology $\mathrm{H}$. Hermeneutic phenomenology and phenomenology: a comparison of historical and methodological considerations. Int J Qual Methods 2003;2:21-35.

59 Villegas M. Hermenéutica Y constructivismo en psicoterapia. Revista de Psicoterapia, 1992: 3. 5-16.

60 Szilas RF. Multiperspective qualitative research methodology for researching work stress. World J Res Rev 2017;5:62-6.

61 Kendall M, Murray SA, Carduff E, et al. Use of multiperspective qualitative interviews to understand patients' and carers' beliefs, experiences, and needs. BMJ 2010;340:196.

62 Braun V, Clarke V. Using thematic analysis in psychology. Qual Res Psychol 2006;3:77-101.

63 Ossó AB, Vigués MP, Fernández RC, et al. To listen, to observe and to understand: bringing back narrative into the health sciences: contributions of qualitative research. 232. Institut Universitari d'Investigació en Atenció Prim ria Jordi Gol (IDIAP Jordi Gol), 2017.

64 Medical Research Council. Implementation science. Available: https://mrc.ukri.org/funding/science-areas/global-health-andinternational-partnerships/applied-global-health-research-board/ourscience-and-contacts/implementation-science/\#_ftnref2 [Accessed 20 Feb 2020].

65 GACD. global alliance for chronic diseases. Available: https://www. gacd.org/research/implementation-science [Accessed 20 Feb 2020].

$66 \mathrm{NIH}$. National cancer Institute. qualitative Methodsational cancer, implementation science, 2018. Available: https://cancercontrol. cancer.gov/IS/docs/NCl-DCCPS-ImplementationScienceWhitePaper.pdf [Accessed 20 Feb 2020].

67 Briones-Vozmediano E. The social construction of fibromyalgia as a health problem from the perspective of policies, professionals, and patients. Glob Health Action 2017;10:1275191.

68 Arnold LM, Crofford LJ, Mease PJ, et al. Patient perspectives on the impact of fibromyalgia. Patient Educ Couns 2008;73:114-20. 